\title{
Enhanced optical force on mutilayered dielectric nanoparticles: Tuning material properties and nature of excitation
}

\author{
Sumit Yadava ${ }^{\mathrm{a}}$, Anita Devi ${ }^{\mathrm{a} \$}$, and Arijit K. De ${ }^{\mathrm{b} *}$ \\ Condensed Phase Dynamics Group, ${ }^{a}$ Department of Physical Sciences and ${ }^{\mathrm{b}}$ Department of \\ Chemical Sciences, Indian Institute of Science Education and Research (IISER) Mohali, \\ Knowledge City, Sector 81, SAS Nagar, Punjab 140306, India. \\ \$Present address: Department of Physics, University of Alberta, 85 Ave, Edmonton, AB T6G \\ 2R3, Canada. \\ *akde@iisermohali.ac.in
}

\begin{abstract}
:
Using dipole approximation, a comparative study of trapping force/potential on different types of dielectric nanoparticles is presented. The trapping force for multilayered nanoparticles, i.e. coreshell-shell type nanoparticles, is found to be enhanced compared with both core-only type and core-shell type nanoparticles. It is shown that an appropriate choice of material and thickness of the middle layer results in tuning the polarizability, thereby playing a vital role in determining the trapping efficiency for core-shell-shell type nanoparticles. Further, the effect of optical nonlinearity under femtosecond pulsed excitation is investigated and it is elucidated that depending on the specific need, the nature of excitation (i.e. pulsed excitation or continuous-wave excitation) can be judiciously chosen. These findings are promised to open up new prospects for controlled nanoscale trapping and manipulation across different fields of nanoscience and nanotechnology.
\end{abstract}

Key words: multilayered nanoparticles, optical trapping, femtosecond pulsed excitation, optical Kerr effect.

\section{Introduction:}

Facile manipulation of nanoparticles can be performed using optical trapping [1] to result in wideranging applications in nanoscience and nanotechnology [2-5]. Optical trapping of dielectric nanoparticles still remained as a challenging task due to the erratic Brownian motion of these particles which increases with decreasing dimensionality. Consequently, to get a stable trap for such particles, very high laser power is used which is otherwise detrimental considering the laserinduced heating, etc effects. As an alternative, repetitive instantaneous trapping through the use of high repetition-rate ultrafast pulsed excitation was envisaged [6]. Subsequently, it was realized 
that under such excitation, nonlinear phenomena, such as the optical Kerr effect (OKE), must be taken into account and OKE was shown to dramatically modulate the trapping efficiency [7]. It was demonstrated that the trapping efficiency is characterized by the height of potential barrier to escape the trapping well, i.e. escape potential [7]. Further studies revealed the usefulness of exploring as well as harnessing optical nonlinearity under pulsed excitation for dielectric [8-10] nanoparticles. Of special interest is to tailor the polarizability of hybrid (i.e. composed of different materials in layered structures) nanoparticles due to their wide-ranging interesting applications, for example, electromagnetic cloaking using multilayered nanoparticles [11] or enhancing spatial resolution without any compromise with the size of particles during constant force measurement in bioconjugated experiment [12-13]. In recent work, we theoretically demonstrated enhanced trapping efficiency for core-shell type nanoparticles over bare which was further shown to be finetuned by optical nonlinearity under pulsed excitation [14]. Also, a reversal (from repulsive to attractive) in optical trapping force under pulsed excitation [14-16]. However, due to the larger tuneability of effective polarizability by the thickness of layers of multilayered nanoparticles are promised to offer better trapping efficiency.

In this article, we present a comparative study of force and potential on core-only or bare type (polystyrene), core-shell (ZnS-polystyrene), and core-shell-shell (ZnS-CdS-polystyrene) type nanoparticles using dipole approximation under both continuous-wave (CW) and pulsed excitation. A significant enhancement in trapping efficiency is observed for core-shell-shell type nanoparticles as compared to bare and core-shell type nanoparticles. It is shown that an appropriate choice of material composition and thickness of the middle layer, i.e. inner shell, can significantly enhance trapping efficiency for the core-shell-shell type nanoparticles, resulting in stable trapping of nanoparticles. Further, we show the effect of optical nonlinearity under pulsed excitation, which can dramatically influence the trapping efficiency of these nanoparticles. Most importantly, the present work shows how a judicious choice of nature of excitation, CW or pulsed excitation, can have certain advantage depending on the specific application.

\section{Mathematical formulation:}

Considering the wide use of polystyrene particles in bio-conjugate experiments [17-18], we choose different types of layered nanoparticles having outer layer as polystyrene. We compare the results for $\mathrm{ZnS}$-polystyrene as core-shell nanoparticle, and $\mathrm{ZnS}$-CdS-polystyrene as core-shell-shell 
nanoparticle with that of core-only type polystyrene nanoparticles. Using a tightly focused Gaussian beam, and corresponding intensity can be written as [7]:

$$
I_{\text {peak } / \text { avg }}(r, z)=\left(\frac{2 P_{\text {peak } / a v g}}{\pi \omega_{0}^{2}}\right) \frac{1}{1+(2 \tilde{z})^{2}} \exp \left[-\frac{2 \tilde{r}^{2}}{1+(2 \tilde{z})^{2}}\right]
$$

where, $\tilde{r}=r / \omega_{0}$ and $\tilde{z}=z / k \omega_{0}^{2}$ are the reduced coordinates; $k=2 \pi n^{w} / \lambda$ is propagation vector inside the medium, $n^{w}$ is the refractive index (RI) of the surrounding medium (water), $\omega_{0}=$ $0.82 \lambda / N A$ is spot-size at focus, $\lambda$ is the wavelength of trapping beam, NA is the numerical aperture of the objective, and $P_{\text {peak/avg }}$ is average power under $\mathrm{CW}$ and peak power under pulsed excitation; here $P_{\text {peak }}=P_{\text {avg }} /_{R R \times \tau} ; R R$ and $\tau$ are the repetition rate and pulse-width, respectively. Along radial direction, only gradient force exists and the trap is always stable due to the restoring nature of this force. However, along the axial direction, gradient and scattering forces act on the particles, and for stable trapping a delicate balance between both the forces is needed. Therefore, the trapping force and potential are calculated along the axial direction only; mathematical expressions for these forces can be written as [7]:

$$
\begin{gathered}
F_{\text {axial,gradient }}(z ; r=0)=-\frac{2 \pi n^{w} a^{3} \alpha}{c} \frac{2 P_{\text {peak } / \text { avg }}}{\pi \omega_{0}^{2}} \frac{8 \tilde{Z} /\left(k \omega_{0}^{2}\right)}{\left(1+4 \tilde{Z}^{2}\right)^{2}} \\
F_{\text {axial,scattering }}(z ; r=0)=\frac{8 \pi n^{w} k^{4} a^{6} \alpha^{2}}{3 c} \frac{2 P_{\text {peak } / \text { avg }}}{\pi \omega_{0}^{2}\left(1+4 \tilde{Z}^{2}\right)}
\end{gathered}
$$

Here, $\mathrm{c}$ is the speed of light, $a$ is the overall radius of the particle, and $\alpha$ is the polarizability per unit volume. The polarizability is a major factor in determining the magnitude as well as the direction of force experienced by the particle. For the bare nanoparticle, the $\alpha$ can be written as:

$$
\alpha=\frac{\alpha^{\prime}}{V}=\frac{\left(n^{p^{2}}-n^{w^{2}}\right)}{\left(n^{p^{2}}+2 n^{w^{2}}\right)}
$$

Here, $n^{p}$ is the RI of the particle and $V$ is $4 \pi n^{w^{2}} \epsilon_{0} a^{3}$. For the core-shell type nanoparticles, $\alpha$ can be calculated as $[9,19]$ :

$$
\alpha=\frac{\alpha \prime}{V}=\frac{\left(n^{s^{2}}-n^{w^{2}}\right)\left(n^{c^{2}}+2 n^{s^{2}}\right)+f^{3}\left(2 n^{s^{2}}+n^{w^{2}}\right)\left(n^{c^{2}}-n^{s^{2}}\right)}{\left(n^{s^{2}}+2 n^{w^{2}}\right)\left(n^{c^{2}}+2 n^{s^{2}}\right)+f^{3}\left(2 n^{s^{2}}-2 n^{w^{2}}\right)\left(n^{c^{2}-n^{s^{2}}}\right)}
$$

where, $n^{c}$, and $n^{s}$ are the RI of core, and shell, respectively. $f=\frac{a_{c}}{a_{s}}$ is the ratio of core radius $\left(a_{c}\right)$ to that of shell $\left(a_{s}\right)$, and $V$ is $4 \pi n^{w^{2}} \epsilon_{0} a_{s}^{3}$. For the core-shell-shell type nanoparticles, $\alpha$ is calculated by considering individual polarizability factors of the core, the inner shell, and shell 2 which can be expressed as [19, 20]: 


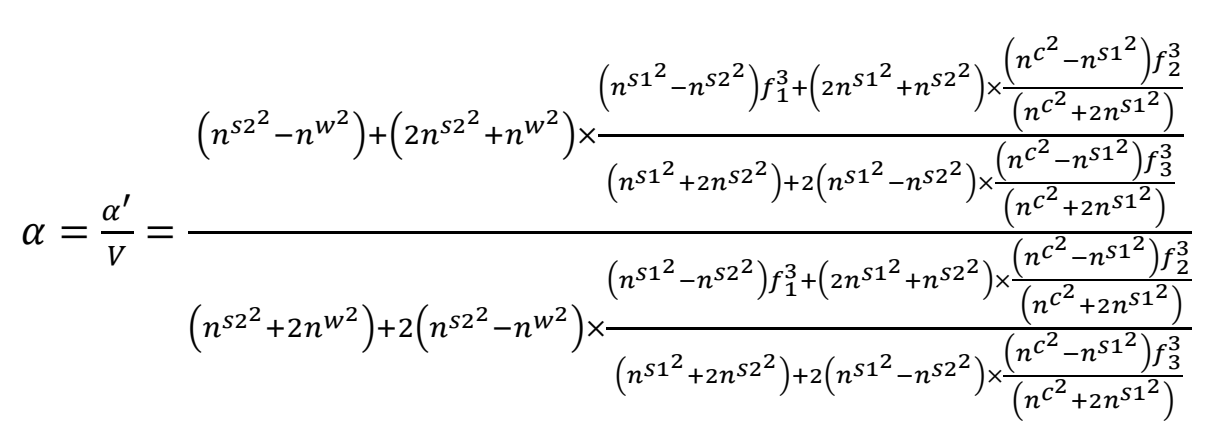

where, $n^{c}, n^{s 1}$, and $n^{s 2}$ are the RI of core, the inner shell, and shell2, respectively. The $f_{1}=$ $\frac{a_{s 1}}{a_{s 2}}, f_{2}=\frac{a_{c}}{a_{s 2}}$, and $f_{3}=\frac{a_{c}}{a_{s 1}}$ are the ratio of the radius of the inner shell $\left(a_{s 1}\right)$ to that of shell2 $\left(a_{s 2}\right)$, the ratio of the radius of core $\left(a_{c}\right)$ to that of shell2 $\left(a_{s 2}\right)$, and the ratio of the radius of core $\left(a_{c}\right)$ to that of the inner shell $\left(a_{s 1}\right)$, respectively, and $V$ is $4 \pi n^{w^{2}} \epsilon_{0} a_{s 2}^{3}$. If $n^{c}=n^{s 1}=n^{s 2}=n^{p}$, we get the $\alpha$ equivalent to core-type nanoparticle, as mentioned in equation $4 \mathrm{a}$.

Further, nonlinear effects (due to optical Kerr effect, OKE) are taken into account in a phenomenological way [21]: $n^{w / c / s 1 / s 2}=n_{0}^{w / c / s 1 / s 2}+n_{2}^{w / c / s 1 / s 2} \times I_{\text {avg/peak }}(r, z)$. Since the NRI for water is very low (refer to table 1), it does not contribute much to the total RI at low average power $(<1 \mathrm{~W})$ and can be neglected for both $\mathrm{CW}$ and pulsed excitation. For particle RI, nonlinearity does not contribute significantly under $\mathrm{CW}$ excitation; however, under pulsed excitation, nonlinear RI contributes significantly to the linear RI of the particle. Thus, in our calculation, we consider:

$$
\begin{gathered}
n^{w / c / s 1 / s 2} \approx n_{0}^{w / c / s 1 / s 2}(\mathrm{CW} \text { excitation) } \\
n^{w} \approx n_{0}^{w} \& n^{c / s 1 / s 2}=n_{0}^{c / s 1 / s 2}+n_{2}^{c / s 1 / s 2} \times I_{\text {peak }}(r, z) \text { (pulsed excitation) }
\end{gathered}
$$

The essential parameters used in the calculations are listed in table 1. The total force (gradient + scattering) is calculated along the axial direction using equations 2 and 3. Under $\mathrm{CW}$ excitation, the force experienced by the particle is equivalent to the average force:

$$
F_{C W} \equiv F_{a v g}
$$

In case of pulsed excitation, the total force is calculated by time averaging over one duty cycle, which can be written as:

$$
\left\langle F_{\text {pulsed }}\right\rangle=\frac{1}{T} \int_{-\tau / 2}^{\tau / 2} F_{\text {peak }} d t
$$

The potential is calculated by numerically integrating the force expressions:

$$
U_{C W}(z ; r=0)=-\int F_{C W}(z ; r=0) d z
$$




$$
U_{\text {pulsed }}(z ; r=0)=-\int\left\langle F_{\text {pulsed }}(z ; r=0)\right\rangle d z
$$

\section{Results and Discussion:}

We numerically simulate the force and potential on hybrid nanoparticles keeping the overall radius of particle fixed as $40 \mathrm{~nm}$ and average power as $100 \mathrm{~mW}$, unless mentioned otherwise.

Figure 1 shows trapping force and potential for the bare, core-shell, and core-shell-shell type nanoparticles under CW excitation. A 20-40 nm ZnS-polystyrene nanoparticle shows a significant enhancement in the force as compared to $40 \mathrm{~nm}$ polystyrene nanoparticle because in $\mathrm{ZnS}$ polystyrene nanoparticle, the RI of the core is greater than that of the shell which results a change in the effective polarizability of the particle. A further enhancement in the force can be evident if a layer of different material is considered in between the $\mathrm{ZnS}$-polystyrene nanoparticle making it three-layered nanoparticles. As shown in figure 1, if a $15 \mathrm{~nm}$ of CdS layer is considered between the $\mathrm{ZnS}$-polystyrene nanoparticle, approximately 2.5 times enhancement in the force maxima is observed as compared to $\mathrm{ZnS}$-polystyrene nanoparticle. This is because the RI of the core and the inner shell is higher than the outer shell, resulting in a significant enhancement in the overall polarizability and consequently enhances the force and the potential. This enhancement in the force is a clear indication of the advantage of using core-shell-shell nanoparticles over core-shell and bare nanoparticles. Such significant enhancement in the force maxima can have potential application in constant force measurements, for example, to determine the transition path dynamics of protein molecules using constant force measurement [12-13]. Core-shell and core-shell-shell type of particles can be potential candidates to enhance the temporal and spatial resolution in such experiments. These results can be evident from the corresponding potential curves as well, as shown in figure 1. As we mentioned earlier, the trap's stability is analyzed from the escape potential [7], marked by a double-sided arrow with corresponding colors. It can be seen that the absolute depth of the potential well increases drastically, whereas escape potential increases slowly. The escape potential is $\sim 8 k_{B} T, \sim 9 k_{B} T$, and $\sim 11 k_{B} T$ for polystyrene, ZnS-polystyrene, and $\mathrm{ZnS}-\mathrm{CdS}$-polystyrene nanoparticles, respectively. Although the change is small, it was shown that even such a small change in escape potential could lead to a significant change in the confinement time of the trapped particle [25]. The noticeable point here is that the nanoparticles of same size can be trapped more efficiently if we use multiple layers instead of bare nanoparticles under similar trapping conditions. 
Based on these results, it appears quite interesting to find the appropriate thickness and nature of the material of the inner shell that corresponds to the most stable trap. In order to estimate that, we now examine the variation of the escape potential with thickness and RI of the inner shell by fixing the overall nanoparticle size as $40 \mathrm{~nm}$ and the core size as $20 \mathrm{~nm}$ (considering polystyrene as the outer layer and $\mathrm{ZnS}$ as the core). Figure 2a shows how escape potential varies against the variation of thickness of the inner shell (while keeping the inner shell material as CdS, i.e. considering ZnS-CdS-polystyrene nanoparticles) from 1 to $19 \mathrm{~nm}$ (i.e. varying $a_{s 1}$ from $21 \mathrm{~nm}$ to $39 \mathrm{~nm}$ while adjusting the thickness of the outer shell to keep the overall particle radius $40 \mathrm{~nm}$ ) under CW excitation. The trapping efficiency varied with the thickness variation of the inner shell, and maximum efficiency is observed for 20-30-40 $\mathrm{nm}\left(a_{c}-a_{s 1}-a_{s 2}\right) \mathrm{ZnS}-\mathrm{CdS}$-polystyrene nanoparticle. Figure $2 \mathrm{~b}$ shows the plot of escape potential against the variation of RI of the inner shell for 20-35-40 nm ZnS-inner shell-polystyrene nanoparticle. With increasing RI, the escape potential increases reach to a maximum, then decreases. While increasing/decreasing the RI of the inner shell, at very high positive/negative RI the scattering force dominates over gradient force, results in destabilizing the trap, and eventually potential become unbound. The value of the RI of the inner shell is $\sim \pm 2$, for which the trap is most stable. Of course, this optimal RI would be different for the different materials' compositions, the thickness of layers, and the nanoparticle's overall size. A crucial finding is that similar behavior is observed for the metamaterials (having negative refractive indices). Thus, different types of materials can be used according to the experimental requirement of the strength of force.

Under pulsed excitation, the OKE is incorporated using equation $5 \mathrm{~b}$; therefore, the nanoparticles' polarizability is inherently dependent on the (average) power of the trapping laser beam. Figure 3 a shows the variation of total effective $\alpha$ against average power for different types of nanoparticles. It can be observed that $\alpha$ increases with average power, but increment is more rapid for bare nanoparticles than the layered nanoparticles. This is because, in different types of nanoparticles, the thickness/radius of the polystyrene layer is $40 \mathrm{~nm}, 20 \mathrm{~nm}$, and $5 \mathrm{~nm}$ in the bare, core-shell, and core-shell-shell type nanoparticles; polystyrene has the highest nonlinear RI among the materials chosen (polystyrene, $\mathrm{ZnS}$ and $\mathrm{CdS}$ ). Therefore, the contribution of nonlinear RI in the total polarizability of hybrid nanoparticles is much dependent on the thickness of the outermost layer. The noticeable point is that at low average power, $\alpha$ (i.e. polarizability per unit volume) is significantly higher for core-shell-shell nanoparticles as compared to bare and core-shell type 
nanoparticles. However, increasing power reverses the case, and at high average power, bare nanoparticles show the dominancy of $\alpha$ over hybrid nanoparticles. Since forces are directly proportional to $\alpha$, enhancement in $\alpha$ results in the enhancement in forces, which implies that at low average power (upto $\sim 60 \mathrm{~mW}$ ) core-shell-shell gives better trapping efficiency than core-shell and bare nanoparticles. The total polarizability depends on the average power and the overall particle size and consequently the escape potential. Figure $3 b$ shows the variation of escape potential for different particle sizes as well as for varying $\alpha$. The contour lines represent the same escape potential for different combinations of particle-size and $\alpha$. For a given $\alpha$, the escape potential increases up to a certain particle-size and then falls off; for example, for $\alpha \approx 1$, the maximum escape potential is for particle size of $25 \mathrm{~nm}$. The maxima in escape potential shifts toward a lower value of $\alpha$ with higher value of particle size. This is because the escape potential decreases with increasing particle size (since scattering force increases more rapidly than the gradient force) as well as increasing polarizability. Therefore, for a given particle-size, trapping efficiency can be tuned by varying the material as well as thickness of the inner shell (which, in effect, changes $\alpha$ ).

We now discuss the effect of optical nonlinearity under pulsed excitation for 20-35-40 nm (ZnS-CdS-polystyrene) nanoparticles. As shown in figure 4, the dragging force experienced by the particle is approximately twice than the $\mathrm{CW}$ excitation (see figure 1 for a comparison); this is also evident from the abosolute depth of the potential well $\left(\sim 55 k_{B} T\right.$ versus $\left.\sim 85 k_{B} T\right)$. However, a decrement in escape potential (and hence in confinement time) is observed as compared to CW excitation at $100 \mathrm{~mW}$ average power $\left(\sim 11 k_{B} T\right.$ versus $\left.\sim 9 k_{B} T\right)$ because the nonlinear RI of polystyrene is higher than $\mathrm{ZnS}$ and $\mathrm{CdS}$. This nonlinear term for polystyrene significantly contributes, rendering the overall RI of polystyrene much higher than $\mathrm{ZnS}$ and $\mathrm{CdS}$ which implies that light will scatter more from the outermost layer. Hence, scattering force dominates over gradient force which results in an unstable trap. From these results, it is evident that pulsed excitation can be either advantageous or disadvatageous over $\mathrm{CW}$ excitation and we can make our choice of nature of excitation judiciously. For example, if we want to use these particles for high force experiment for a short duration of trapping, we can take the advantage of impulsive dragging force under pulsed excitation. On the other hand, if we need the particles to be confined for a longer time, $\mathrm{CW}$ excitation is more suitable. 
Now, to determine any dependence on the thickness of the inner shell (CdS layer) for which pulsed excitation may be more advantageous over $\mathrm{CW}$ excitation, we study the variation of escape potential with thickness of the inner shell which varies from $1 \mathrm{~nm}$ to $19 \mathrm{~nm}$, as shown in figure $5 \mathrm{a}$. Quite interestingly, at $100 \mathrm{~mW}$ average power, increasing thickness of CdS layer decreases the escape potential monotonically, however, at $150 \mathrm{~mW}$ average power, the case is reversed. Thus, one can readily identify the advantage of pulsed excitation over $\mathrm{CW}$ excitation and choose the type of excitation depending on the specific need (high-force experiments versus long confinement time exeriments). This advantage is even more prominent for the CdS layer ( $a_{s 1}$ greater than $30 \mathrm{~nm}$ ), at high average power, as shown in figure $5 \mathrm{~b}$. Figure $5 \mathrm{c}$ shows escape potential against RI of the inner shell $\left(n_{0}^{s 1}\right)$ at different average power under pulsed excitation. Please note that under pulsed excitation, the nonlinear RI of the inner shell is kept constant; hence, RI of the inner shell can be written as: $n^{s 1}=n_{0}^{s 1}+n_{2}^{s 1} \times I_{\text {peak }}(r, z)=n_{0}^{s 1}+1.68 \times 10^{-18} \times I_{\text {peak }}(r, z)$; here we consider $n_{0}^{s 1}$ as variable. At $50 \mathrm{~mW}$ and $100 \mathrm{~mW}$ average powers, the trend of the curve is very similar to $\mathrm{CW}$ excitation (figure $2 \mathrm{~b}$ ), however, the value of $n_{0}^{s 1}$ for which the trap is most stable shifts towards lower $\left|n_{0}^{s 1}\right|$. Further increase in average power decreases the value of $n_{0}^{s 1}$ corresponding to the most stable trap, and after a certain average power $(\sim 500 \mathrm{~mW})$, both the peaks merge into one peak. The nonlinear part of the RI of the inner shell $\left(1.68 \times 10^{-18} \times I_{\text {peak }}\right)$ increases with power, and the values are $0.0205,0.0411,0.2058$, and 0.4116 at 50,100, 500, and $1000 \mathrm{~mW}$ at average power, respectively. The effective $\alpha$ increases with the increasing contribution of nonlinear RIs of different layers with power; therefore, the optimal value of $\alpha$ corresponding to the most stable trap requires a less contribution from linear RI due to which the peaks shifts towards the lower value of $\left|n_{0}^{s 1}\right|$ with power and eventually decreases close to zero (marked by merging of peaks), as shown in figure $5 \mathrm{c}$. From this, it is evident that by taking advantage of OKE, we can trap those particles that have RI very less than the surrounding medium; such particles cannot be trapped using CW excitation under similar conditions. In contrast, high RI (both linear and nonlinear) particles cannot be trapped under pulsed excitation at high average power because scattering force dominant over the gradient force. However, under CW excitation, a few high RI particles can be trapped with better trapping efficiencies under similar conditions [9].

Further, we examine the trapping of hollow core-shell-shell type nanoparticles by fixing the nanoparticle's overall size as $35 \mathrm{~nm}$, considering many practical applications of hollow nanoparticles, for example in targeted drug delivery [26]. Under CW excitation, 30-35 nm hollow- 
polystyrene nanoparticle experiences a repulsive force, as shown in figure 6. However, the presence of $2 \mathrm{~nm} \mathrm{CdS}$ layer in-between the hollow-polystyrene nanoparticle allows the particle to experience an attractive force under similar excitation conditions. As mentioned earlier, the reversal nature of force (from repulsive to attractive) was shown for hollow-core nanoparticles upon changing the excitation from CW to pulsed (owing to the effect of OKE) [14-16]. In contrast, for a hollow core-shell-shell type nanoparticles, even under CW excitation such a reversal in the nature of the force is observed; note that similar reversal under CW excitation was also observed for hollow-core type nanoparticles by tuning the thickness of the material [16]. Under pulsed excitation, owing to the OKE, both hollow-polystyrene and hollow-CdS-polystyrene nanoparticles experience an attractive force. Also, an enhancement in force is observed which is evident from the potential curves as well.

\section{Conclusion:}

In summary, the results presented in this article show that both CW and pulsed excitations have their own advantages and disadvantages, depending on the material properties, particle-size and excitation parameters (average power, etc) and nature of excitation (pulsed versus $\mathrm{CW}$ ). An appropriate choice of these parameters may enhances the pondermotive optical force or enhance the duration of trapping and the effects are manifested to different extent depending on the type of

particles (bare versus multilayered type). These results are promised to break new grounds through controlled nanoscale optical manipulation.

\section{Acknowledgments:}

This research was supported by Early Career Research Award from SERB, DST (Grant No: ECR/2016/000467) and by Start-up Grant from IISER Mohali. SY and AD acknowledge UGC and IISER Mohali, respectively, for Graduate Fellowships.

\section{Contributions:}

SY and AD contributed equally in this work.

\section{Declaration:}

The authors declare no competeing interest. 


\section{References:}

1. A. Ashkin, J. M. Dziedzic, J. E. Bjorkholm, and S. Chu, "Observation of a single-beam gradient force optical trap for dielectric particles," Opt. Lett. 11, 288-290 (1986).

2. K. C. Neuman, and S. M. Block, “Optical trapping,” Rev. Sci. Instrum. 75, 2787-2809 (2004).

3. A. Ashkin, "Optical trapping and manipulation of neutral particles using lasers: A reprint volume with commentaries," World Scientific (2006).

4. J. R. Moffitt, Y. R. Chemla, S. B. Smith, and C. Bustamante, "Recent advances in optical tweezers," Annu. Rev. Biochem. 77, 205-228 (2008).

5. D. Gao, W. Ding, M. Nieto-Vesperinas, X. Ding, M. Rahman, T. Zhang, C. Lim, and CW. Qiu, "Optical manipulation from the microscale to the nanoscale: fundamentals, advances and prospects," Light Sci. Appl. 6, e17039 (2017).

6. D. Roy, D. Goswami, and A. K. De, "Exploring the physics of efficient optical trapping of dielectric nano-particles with ultrafast pulsed excitation," Appl. Opt. 54(23), 7002-7006 (2015).

7. A. Devi, and A. K. De, "Theoretical investigation on nonlinear optical effect in laser trapping of dielectric nanoparticles with ultrafast pulsed excitation,” Opt. Express 24, 21485-21496 (2016).

8. A. Devi, and A. K. De, "Theoretical estimation of nonlinear optical force on dielectric spherical particles of arbitrary size under femtosecond pulsed excitation," Phys. Rev. A 96, 023856 (2017).

9. A. Devi, and A. K. De, "Generalized description of the nonlinear optical force in laser trapping of dielectric nanoparticles," Phys. Rev. Res. 2 (4), 043378 (2020).

10. A. Devi, and A. K. De, "Unified treatment of nonlinear optical force in laser trapping of dielectric particles of varying sizes," Phys. Rev. Res. 3, 033074 (2021).

11. S. Batool, M. Nisar, F. Frezza and F. Mangini, "Cloaking Using the Anisotropic Multilayer Sphere," Photonics 7(3), 52 (2020).

12. M. Capitanio and F. S. Pavone, "Interrogating Biology with Force: Single Molecule HighResolution Measurements with Optical Tweezers,” Biophys. J. 105, 1293-1303 (2013).

13. K. C. Neuman and A. Nagy, "Single-molecule force spectroscopy: optical tweezers, magnetic tweezers and atomic force microscopy," Nat. Methods 5(6), 491-505 (2008). 
14. A. Devi, S. S. Nair, S. Yadav, and A. K. De, "Controlling optical trapping of metaldielectric hybrid nanoparticles under ultrafast pulsed excitation: a theoretical investigation," Nanoscale Adv. 3(11), 3288-3297 (2021).

15. A. Devi, and A. K. De, "Harnessing optical nonlinearity to control reversal of trapping force: A theoretical investigation,” J. Opt. 21, 065502 (2019).

16. A. Devi, and A. K. De, "Generalized Lorenz-Mie theory for the reversal of optical force in a nonlinear laser trap,” Phys. Rev. A 102, 023509 (2020).

17. K. Chatterjee, S. Sarkar, K. J. Rao, and S. Paria, "Core/shell nanoparticles in biomedical applications," Adv. Colloid Interface Sci. 209, 8-39 (2014).

18. S. Komoto, T. Nagai, R. Takao, K. Ushiro, M. Matsumoto, T. Shoji, D. P. Linklater, S. Juodkazis, and Y. Tsuboi, "Optical Trapping of Polystyrene Nanoparticles on Black Silicon: Implications for Trapping and Studying Bacteria and Viruses," ACS Appl. Nano Mater. 3(10), 9831-9841, (2020).

19. H. C. V. D. Hulst, "Light Scattering by Small Particles," (Dover, New York, 1981).

20. A. Sihvola, and I.V. Lindell, "Transmission Line Analogy for Calculating the Effective Permittivity of Mixtures with Spherical Multilayer Scatterers," J. Electromagn. Waves Appl. 2(8), 741-756 (1988).

21. R. W. Boyd, “Nonlinear Optics,” Academic Press, San Diego (1992).

22. http://refractiveindex.info/.

23. M. Jahja, "On nonlinear optical constants of polystyrene," International Symposium on Modern Optics and Its Applications-2011 (2011).

24. T. D. Krauss, and F. W. Wise, "Femtosecond measurement of nonlinear absorption and refraction in CdS, ZnSe, and ZnS," Appl. Phys. Lett. 65, 1739 (1994).

25. A. Devi, S. Yadav, and A. K. De, "Dynamics of a dielectric microsphere inside a nonlinear laser trap," Appl. Phys. Lett. 117(16), 161102 (2020).

26. D. He, S. Wang, L. Lei, Z. Hou, P. Shang, X. He, and H. Nie, "Core-shell particles for controllable release of drug," Chem. Eng. Sci. 125, 108-120 (2015). 


\section{Tables and captions:}

\begin{tabular}{|c|c|c|}
\hline Parameters used & Symbol & Value/Expression \\
\hline $\begin{array}{c}\text { Central wavelength } \\
\text { Speed of light }\end{array}$ & $\lambda$ & $800 \mathrm{~nm}$ \\
\hline Repetition rate & $f$ & $3 \times 10^{8} \mathrm{~m} / \mathrm{s}$ \\
\hline Pulse width & $\tau$ & $76 \mathrm{MHz}$ \\
\hline Numerical aperture & $\mathrm{NA}$ & $120 \mathrm{fs}$ \\
\hline Linear RI of the medium [22] & $n_{0}^{w}$ & 1.4 \\
\hline $2^{\text {nd }}$ order NRI of water [7] & $n_{2}^{w}$ & $2.7 \times 10^{-20} \mathrm{~m}^{2} / \mathrm{W}$ \\
\hline Linear RI of polystyrene [22] & $n_{0}^{s 2}$ & 1.578 \\
\hline $2^{\text {nd }}$ order NRI of polystyrene [23] & $n_{2}^{s 2}$ & $5.9 \times 10^{-17} \mathrm{~m}^{2} / \mathrm{W}$ \\
\hline Linear RI of CdS [22] & $n_{0}^{s 1}$ & 2.386 \\
\hline $2^{\text {nd }}$ order NRI of CdS [24] & $n_{2}^{s 1}$ & $1.68 \times 10^{-18} \mathrm{~m}^{2} / \mathrm{W}$ \\
\hline Linear RI of ZnS [22] & $n_{0}^{c}$ & 2.313 \\
\hline $2^{\text {nd }}$ order NRI of ZnS [24] & $n_{2}^{c}$ & $3.8 \times 10^{-19} \mathrm{~m}^{2} / W$ \\
\hline
\end{tabular}

Table 1: List of the parameters used in numerical calculations. NRI: nonlinear refractive index. 
Figures and captions:

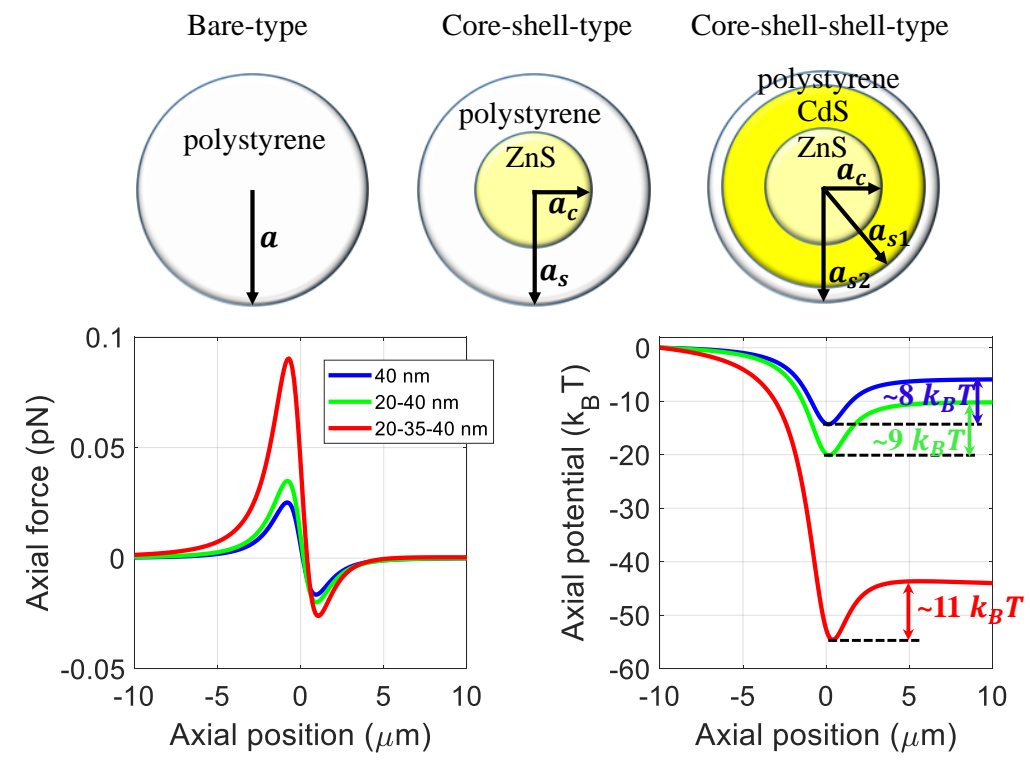

Figure 1. Trapping force and potential for polystyrene/ZnS-polystyrene/ZnS-CdS-polystyrene nanoparticles under $\mathrm{CW}$ excitation at $100 \mathrm{~mW}$ average power. Color: blue/green/red curve represents the polystyrene/ZnSpolystyrene/ZnS-CdS-polystyrene nanoparticles, respectively.
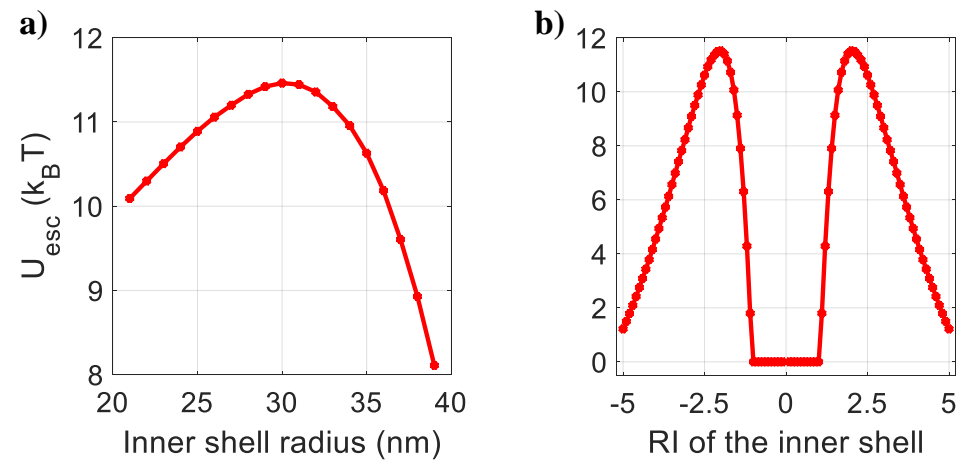

Figure 2. Variation of escape potential with a) inner shell radius and b) RI of the inner shell for core-shell-shell type nanoparticles under $\mathrm{CW}$ excitation at $100 \mathrm{~mW}$ average power. 
a)

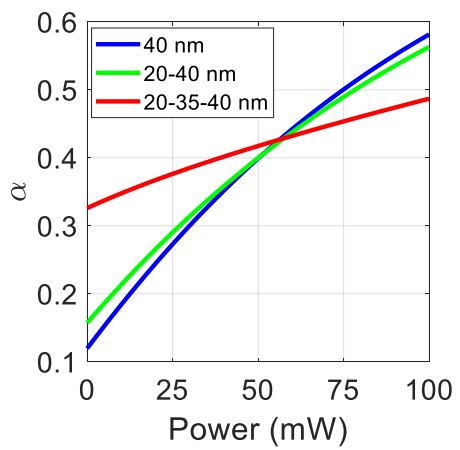

b)

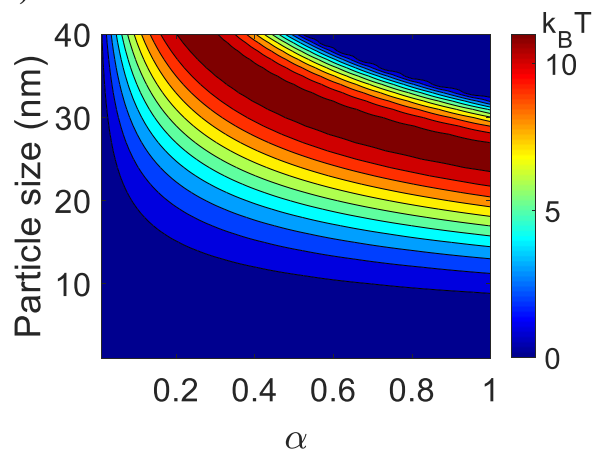

Figure 3. Variation of a) polarizability per unit volume against average power for all three types of nanoparticles at a fixed $40 \mathrm{~nm}$ overall size under pulsed excitation. b) Variation of escape potential with polarizability per unit volume and particle size at $100 \mathrm{~mW}$ average power. Color: blue/green/red curve represents the polystyrene/ZnS-

polystyrene/ZnS-CdS-polystyrene nanoparticles, respectively.
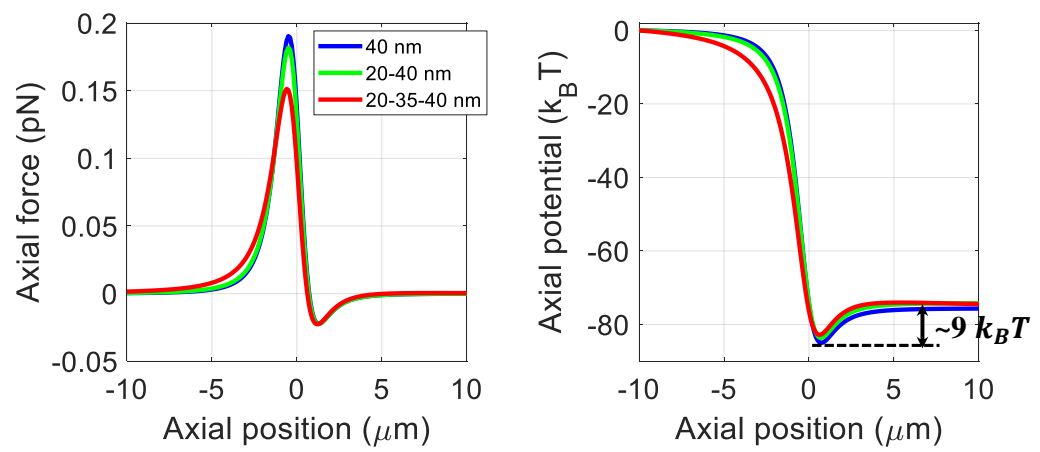

Figure 4. Trapping force or potential for polystyrene/ZnS-polystyrene/ZnS-CdS-polystyrene nanoparticles under pulsed excitation at $100 \mathrm{~mW}$ average power. Color: blue/green/red curve represents the polystyrene/ZnSpolystyrene/ZnS-CdS-polystyrene nanoparticles, respectively.

a)

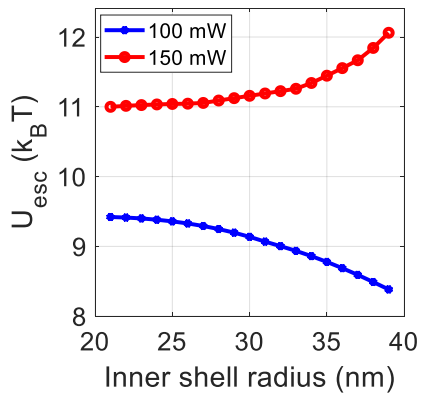

b)

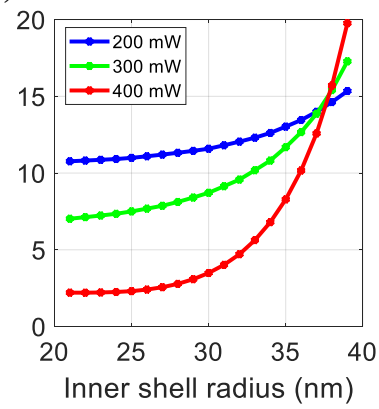

c)

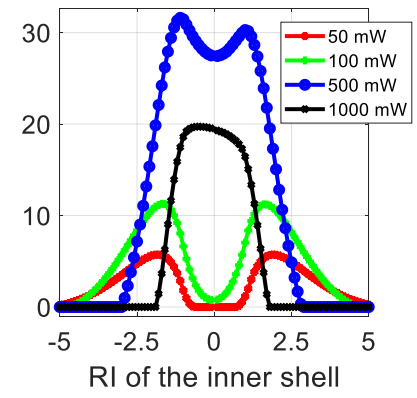

Figure 5. Plots of escape potential against a-b) variation of the inner shell radius and c) variation of RI of the inner shell at different average powers under pulsed excitation. 

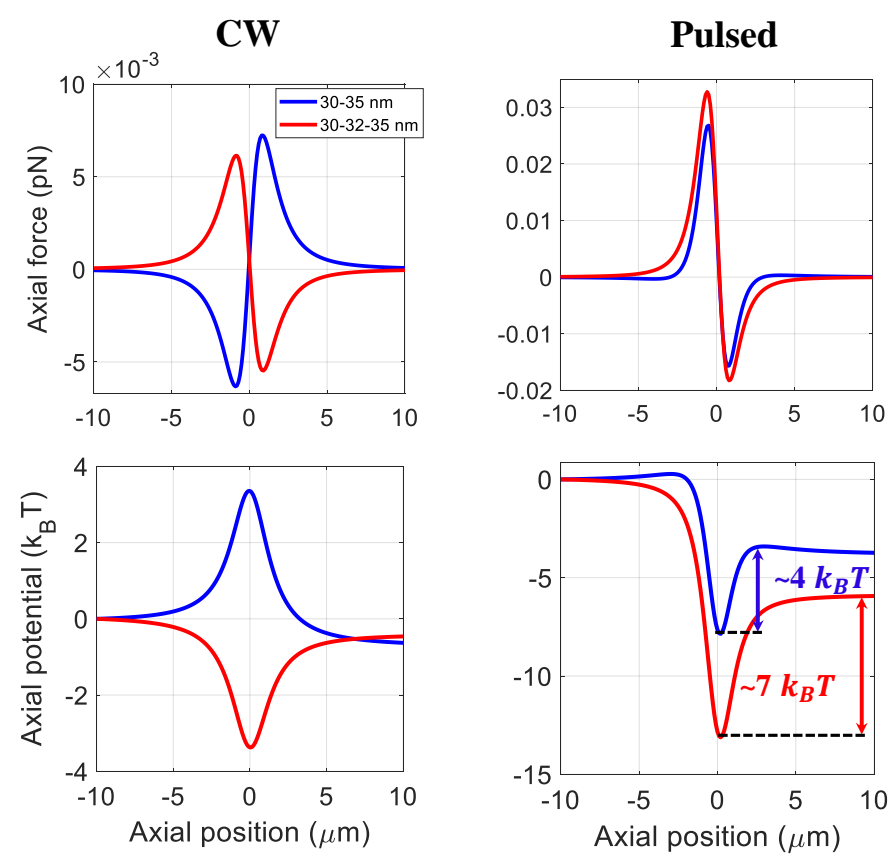

Figure 6. Trapping force and potential for hollow core-shell and hollow core-shell-shell type nanoparticles at 100 $\mathrm{mW}$ average power under $\mathrm{CW}$ and pulsed excitation. 